\title{
Movilización legal: una estrategia sindical con efectos ambivalentes*
}

\author{
Legal Mobilization: a union strategy with ambivalent effects
}

\author{
Francisca Gutiérrez Crocco** e Ignacio Gutiérrez Crocco***
}

\begin{abstract}
Resumen: Desde la recuperación de la democracia en 1990, la movilización legal se ha vuelto una estrategia clave en el repertorio de acción del movimiento sindical chileno. Los sindicatos recurren cada vez más a la Dirección del Trabajo o los tribunales para contestar el poder de los empresarios y presionar al Estado para lograr cambios en el orden laboral heredado de la dictadura. En base a entrevistas a distintos actores, este artículo evalúa los efectos de este fenómeno para los trabajadores. Defiende que la movilización legal ha permitido a los sindicatos ganar ciertos derechos y ampliar su margen de maniobra en las empresas. Sin embargo, no ha generado cambios sustanciales en el orden laboral chileno. El artículo identifica dos causas de esto: 1) los efectos no deseados del uso de esta estrategia en la fuerza de la organización sindical; 2) los cambios en la orientación de la DT y los tribunales.
\end{abstract}

Palabras clave: Judicialización; Movilización legal; Dirección del Trabajo; Tribunales; Sindicatos; Movimiento laboral; Trabajadores

\begin{abstract}
Since the recovery of democracy in 1990, legal mobilization has become a key strategy for the Chilean union movement. Trade unions increasingly turn to the Labor Department or the courts for challenging the employers' power and putting pressure over the State to make changes on the labor order. Based on interviews with different labor actors, this article evaluates the effects of this phenomenon for workers. It argues that legal mobilization has allowed unions to gain certain rights and expand their margin of action in companies. However, this strategy has not produced substantial changes in the inherited labor order. The article identifies two causes of this: 1) the undesirable effects of the use of this strategy on the strength of the trade union organization; 2) changes in the orientation of the Labor Department and the courts.
\end{abstract}

Keywords: Judicialization; Legal mobilization; Labor Department; Courts; Unions; Labor movement; Workers

\footnotetext{
* La recolección de algunos de los datos utilizados y la redacción de este artículo han sido posible gracias al financiamiento de FONDAP Nº15130009, PAI No 79140069 y FONDECYT Nº11150217.

** Chilena, autora principal. PhD en Sociología, Profesora Asistente en Escuela de Economía y Negocios en Universidad Alberto Hurtado (UAH); Investigadora Adjunta en Centro de Estudios de Conflicto y Cohesión Social (COES). Correo electrónico: fgutierrez@uahurtado.cl.

**** Chileno, autor secundario. Psicólogo, Asistente de investigación en Centro de Investigación para la Gestión Integrada de desastres Naturales (CIGIDEN). Correo electrónico: iagutierrezcrocco@ gmail.com.
} 
Recibido: 08/03/2017

Aceptado:19/06/2017

\section{Introducción}

Desde los años 1990, se han multiplicado los estudios de caso que muestran cómo movimientos sociales hacen uso de los tribunales para impulsar procesos de democratización o intentar generar cambios sociales que consoliden las democracias existentes ${ }^{1}$. Utilizan los procesos judiciales para impugnar iniciativas de los otros poderes del Estado o para forzar interpretaciones que expandan el sentido de la ley a favor de los grupos menos poderosos. Los estudiosos sobre movimientos sociales han acuñado el término de movilización legal para referirse a este tipo de estrategia ${ }^{2}$. Sin embargo, la inclinación de los movimientos sociales a privilegiar la movilización legal respecto de otras formas de acción política, tales como la protesta o la manifestación, ha suscitado una nutrida polémica. ¿En qué medida la movilización legal puede promover un cambio social significativo?

En el ámbito específico de los estudios laborales, este debate ha generado escaso interés, pese a la creciente importancia de la movilización legal para los sindicatos. En efecto, existe evidencia que esta estrategia tiene cada vez un peso mayor en el repertorio de acción de las organizaciones de trabajadores, tanto en países desarrollados ${ }^{3}$, como emergentes ${ }^{4}$. Chile es un caso paradigmático de esta tendencia. Como demostraremos más adelante, las restricciones impuestas por las reformas neoliberales de los años 1980 a la negociación colectiva y a la huelga, obligaron a los sindicatos a buscar nuevas vías para defender los intereses de los trabajadores frente a la empresa y el Estado. Denunciar a la empresa ante la Dirección del Trabajo (DT) y/o los tribunales se transformó, desde la década de 1990, en una de las estrategias más recurrentes para lograr estos objetivos. A pesar de la visibilidad e importancia de estos cambios, no existen estudios que discutan las consecuencias de este fenómeno para los trabajadores en Chile.

1 Epp, Charles R. The Rights Revolution: Lawyers, Activists, and Supreme Courts in Comparative Perspective, $1^{\mathrm{a}}$ ed, Chicago: University Of Chicago Press, 1998; McCAnN, Michael. Rights at Work: Pay Equity Reform and the Politics of Legal Mobilization, Chicago: University of Chicago Press, 1994; BursteIn, Paul. "Legal Mobilization as a Social Movement Tactic: The Struggle for Equal Employment Opportunity", American Journal of Sociology 96:5, 1991, p.1201-25.

2 BURSTEIN, 1991, op. cit.

3 Roomkin, Myron. "A Quantitative Study of Unfair Labor Practice Cases”, Industrial and Labor Relations Review 34:2, 1981, p.245-256; MCCAMmON, Holly J.. "Labor's Legal Mobilization Why and When Do Workers File Unfair Labor Practices?", Work and Occupations 28:2, 2001, p.143-75

4 Chen, Feng. "Legal Mobilization by Trade Unions: The Case of Shanghai", The China Journal, 52: Julliet, 2004, p.27-45; De FAZIO, Federico Leandro. "Judicialización de la protesta sindical en Argentina: El control de constitucionalidad como espacio de participación alternativo", Gaceta Laboral 19:1, 2013. 
A partir de entrevistas a distintos actores del mundo laboral, este artículo discute los efectos que ha tenido para los trabajadores el creciente uso de la acción legal por parte del movimiento sindical chileno. Se espera que esta discusión aporte al debate sobre las limitaciones y ventajas que tiene el uso de la movilización legal como medio para consolidar la democracia. En este caso, se considera el recurso a la DT como una forma de movilización legal complementaria a la que se lleva a cabo en tribunales. Si bien la DT es una agencia administrativa que depende del Poder ejecutivo, cumple un rol clave en la vigilancia y sanción de las infracciones a la ley laboral. Los trabajadores pueden denunciar a las empresas ante la DT en lugar de los tribunales, incluso en algunos casos, es un requisito de admisibilidad de la demanda ante tribunales. Asimismo, la DT tiene la potestad de interpretar la ley para ajustarla a los casos concretos y, por ende, puede generar una ampliación o restricción de los derechos de trabajadores, aun cuando este cambio no es vinculante para los tribunales. Con el fin de facilitar la lectura del texto, se hablará de "instituciones arbitradoras" (IAs) cuando se haga referencia a ambas instituciones en conjunto, la DT y los tribunales.

El artículo está dividido en cuatro secciones. En la primera, se resume la discusión que ha existido en torno al tema de la movilización legal en la literatura sobre movimientos sociales. En la segunda, se pone en contexto la discusión, examinando cómo la movilización legal se ha transformado en una estrategia central en el repertorio de acción de los sindicatos chilenos. En la tercera sección, se detalla la metodología utilizada para recolectar y analizar los datos sobre Chile que se utilizan en el texto. Por último, en la cuarta sección, se presentan los resultados del análisis de las entrevistas.

\section{Movilización Legal ¿un medio para el cambio social?}

La expansión de la influencia del Poder judicial en la toma de decisiones políticas y en la regulación de la vida social en general, ha sido objeto de un extenso debate en las ciencias políticas. Durante mucho tiempo, se asumió implícitamente que este proceso de "judicialización" era el producto de la presión ejercida por los mismos jueces. Los estudiosos de los movimientos sociales demostraron que este no era siempre el caso y comenzaron a estudiar la intervención de los jueces como el resultado de una estrategia de la sociedad civil para generar cambios sociales ${ }^{5}$. Se acuñó el concepto de "movilización legal" para referir a este último tipo de acciones.

La literatura empírica, ha dado cuenta de la existencia de distintos tipos de movilización legal. Puede tratarse de acciones ante cortes constitucionales que se involucran en el diseño e implementación de políticas públicas como en Estados Unidos, o de acciones que se presentan ante cortes que tienen la función de controlar la supremacía constitucional de las iniciativas de los otros poderes, como en América Latina ${ }^{6}$. En estos dos casos, los

5 MCCANN, 1994, op. cit.; EPP, 1998, op. cit.

6 SiEder, Rachel, Line Schjolden, y Adam Angell (eds). The Judicialization of Politics in Latin America, New York: 
movimientos sociales buscan el cambio social a través de la incidencia directa en el proceso político. En un sentido más general, la movilización legal puede referirse a las acciones que permiten a las organizaciones de la sociedad civil aclarar o ampliar la definición de una norma. De esta manera, normativas que benefician en principio el poder de la élite pueden tornarse a favor de grupos minoritarios o menos poderosos ${ }^{7}$. A diferencia de lo que sucede en el sistema anglosajón donde las sentencias judiciales tienen en sí mismas valor de ley, en un sistema continental como el chileno, la nueva interpretación de la ley tiene un efecto indirecto en la ley. Si bien no es vinculante, abre la posibilidad que las organizaciones recurran a las IAs para impugnar comportamientos de los grupos poderosos que antes no eran sancionados porque la norma era ambigua al respecto y que presionen a las autoridades para que modifiquen la ley en coherencia con el nuevo sentido otorgado. Al poder que otorga este cambio de interpretación en un sistema como el chileno lo llamaremos en adelante "derecho de hecho".

Sin embargo, la capacidad de la movilización legal de promover cambios sociales ha sido ampliamente discutida. Según revisiones recientes de la literatura este debate ha tendido a enmarcarse en dos campos: el campo de derechos y el simbólico-estratégico ${ }^{8}$, habiendo argumentos optimistas y pesimistas en ambos campos del debate.

En el campo de derechos, el argumento optimista subraya que la movilización legal puede ser una estrategia atractiva para los grupos en desventaja política, en cuanto el Poder judicial proporciona una vía abierta y accesible para exigir cambios en los derechos. Aun cuando los objetivos de un movimiento no cuenten con apoyo de los grupos con poder político o este movimiento carezca de los recursos necesarios para promover su causa por la vía política, las instancias judiciales generalmente son independientes y están obligadas a considerar las demandas de este grupo ${ }^{9}$.

Un argumento menos optimista en este campo pone el acento en los obstáculos que encuentra a menudo la movilización legal. Por un lado, se ha planteado que el Poder judicial tiene pocas posibilidades de producir un cambio significativo en los derechos por sí solo, pues depende de otros factores, requiriendo de contextos específicos y la acción de otros poderes ${ }^{10}$. En la misma línea, se ha observado que los jueces no siempre hacen uso de su facultad interpretativa en favor de los derechos de los grupos más débiles. Los tribunales

Palgrave Macmillan, 2005.

7 Shapiro, Martin. Courts: A Comparative and Political Analysis, Chicago: Univ. of Chicago Press, 1981; CiCHOwsKi, Rachel A. "Women's Rights, the European Court, and Supranational Constitutionalism", Low \& Society Review, 38:3, 2004, p.489-513.

8 Albiston, Catherine. “The Dark Side of Litigation as a Social Movement Strategy", Iowa Law Review Bulletin, 96:941, 2011, p.61-77.

9 HANDLER, Joel F. Social Movements and the Legal System: A Theory of Law Reform and Social Change. New York: Academic, 1978; NeJAime, Douglas. "The legal mobilzation dilemma”, Emory Law Journal, 61:32, 2011, p.663-735.

10 Rosenberg, Gerald. The Hollow Hope: Can Courts Bring About Social Change?, Chicago: University of Chicago Press, 1991; ScheIngold, Stuart. The Politics of Rights, Lawyers, Public Policy, and Political Change, New Haven, CT: Yale Univ. Press, 1974. 
pueden identificarse con los intereses de la élite y actuar en función del statu quo ${ }^{11}$. En este sentido, la existencia de una infraestructura adecuada para la denuncia y de grupos capaces de empujar una causa en estas instancias judiciales no siempre bastan para lograr lo que Charles Epp ${ }^{12}$ denomina una "revolución de derechos". Para que este cambio se produzca también es necesario que los miembros del Poder judicial sean favorables a ese cambio. Un ejemplo que ilustra lo anterior es lo que sucedió en Chile al término de la dictadura militar. Sólo a fines de los 1990, la orientación de la Corte Suprema cambió en materia de derechos humanos y permitió la sanción de los crímenes perpetrados por el Estado durante la dictadura, pero siguió siendo conservadora en materias como derechos reproductivos, divorcio, libertad de expresión, conflicto mapuche, entre otros ${ }^{13}$. Por otro lado, se postula que la movilización legal puede fomentar una atomización de las demandas que debilita en último término al movimiento. La accesibilidad de las acciones judiciales facilita que individuos puedan realizar sus propias demandas sin requerir que represente a un grupo, lo que puede producir que demandas individuales resulten en decisiones judiciales que afectan a otros miembros del movimiento y restrinjan el avance del movimiento general ${ }^{14}$.

El debate en el campo simbólico-estratégico refiere a los efectos indirectos de movilización legal sobre el movimiento mismo u otros grupos implicados. La visión optimista subraya que los movimientos sociales no sólo pueden beneficiarse por obtener sentencias favorables a su causa al emprender una campaña judicial. Esta acción puede ayudarlos a dar forma y visibilidad a sus demandas, ganar adherentes al movimiento, crear aliados, atraer recursos financieros o dejar un legado ${ }^{15}$. Asimismo, las campañas legales pueden ayudar al movimiento a mejorar su imagen ante la sociedad. Por ejemplo, Chen ${ }^{16}$ demuestra que los sindicatos chinos se han vuelto hacia una estrategia judicial y que, de esta manera, han logrado reposicionarse como un actor que defiende a los trabajadores, a pesar de la estrecha relación que los ata al gobierno comunista y que les impide jugar un rol frontalmente disruptivo ${ }^{17}$.

No obstante, la movilización legal también puede perjudicar indirectamente la causa de quienes persiguen un cambio social. Una mirada menos optimista ha observado que la exclusiva concentración en este tipo de acciones puede tener efectos no deseados para las organizaciones o movimientos sociales. Puede producir una dificultad para movilizar más activamente a las bases con el fin de lograr cambios más sustanciales. Una conclusión de este tipo arrojó el estudio de William Forbath sobre el movimiento obrero norteamericano a

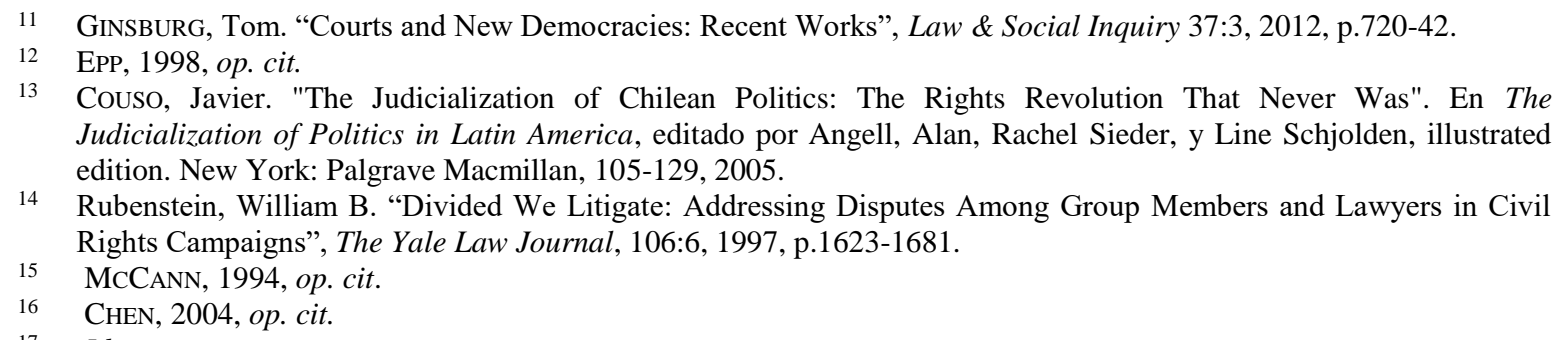


fines del siglo XIX, el cual por concentrarse en una estrategia judicial y dejar de lado la movilización política, habría perdido radicalidad $^{18}$. Por otro lado, la movilización legal puede producir una pérdida de autonomía. La dependencia de los movimientos hacia las instituciones judiciales puede producir que sus demandas se limiten al marco judicial mismo que buscan cambiar, legitimando el sistema y resignificando sus demandas a éste ${ }^{19}$.

En suma, la literatura sobre movilización legal parece coincidir en que los efectos de esta estrategia y su capacidad de generar cambios sociales dependen, en gran medida, del contexto en que se desenvuelve. La evaluación debe tomar en cuenta las particularidades de los actores que llevan a cabo estas acciones, el sistema judicial y el campo político, entre otros elementos.

\section{La movilización legal en el repertorio de acción de los sindicatos}

El golpe militar de 1973 marcó el inicio de un periodo de grandes transformaciones en el repertorio de acción del movimiento sindical chileno. Tras un periodo de fuerte represión en contra de los dirigentes más politizados, el régimen de Augusto Pinochet promulgó una serie de leyes que transformarían las reglas del juego a favor de los empresarios. Con el fin de adaptar la legislación laboral a una economía de mercado, el llamado Plan Laboral (1979) eliminó varios de los derechos conquistados por el movimiento laboral durante las décadas anteriores. Los consejeros económicos del régimen creían que la inversión y la creación de empleos se veía dañada por la excesiva regulación, y que los costos laborales externos al salario contribuían a aumentar innecesariamente los costos laborales. Consecuentemente, el Plan limitó la negociación colectiva a la empresa y redujo el alcance de este proceso al ajuste salarial. Asimismo, autorizó a los trabajadores a organizarse para negociar sin la representación de un sindicato a través de los llamados "grupos de negociación". Las restricciones al derecho a huelga fueron aún más severas. El Plan desincentivó a los sindicatos a recurrir a una huelga e hizo muy difícil que las organizaciones que desafiaban estas barreras ganaran esta campaña. Por ejemplo, permitió a los empleadores contratar trabajadores de reemplazo durante la huelga. Asimismo, si los trabajadores recurrían a una huelga, sus puestos de trabajo sólo estaban asegurados por 59 días, después de lo cual el empleador podía despedirlos sin necesidad de justificar su decisión.

La recuperación de la democracia en 1990 y el triunfo de la Concertación de Partidos por la Democracia (en adelante "Concertación") creó grandes expectativas en el mundo sindical. Dada su participación en el movimiento que permitió el término de la dictadura y los lazos con las nuevas autoridades, los líderes de la Central Unitaria de Trabajadores (CUT) confiaron que la recuperación de los derechos sindicales perdidos sería cuestión de tiempo.

18 Forbath, William. "Law and the shaping of the american labor movement", citado en ALBISTON, 2011, p.75, op. cit.

19 Albiston, 2011, op. cit. 
Sin embargo, las reformas laborales que siguieron, la primera en 1991 y la segunda el $2001^{20}$, no cumplieron con las expectativas de los dirigentes. Si bien la Concertación dio ciertas concesiones a los sindicatos como la reducción del quorum necesario para formar sindicatos en las empresas pequeñas o la simplificación de los procedimientos para la organización de los trabajadores temporales, los avances en materia de derechos colectivos fueron escasos. Las reformas no ampliaron considerablemente las temáticas susceptibles de ser negociadas ni aprobaron la negociación de rama. Tampoco eliminaron los "grupos de negociación" o el derecho de los empleadores a reemplazar a los trabajadores en huelga. La legislación conservó en este ámbito el espíritu del Plan Laboral ${ }^{21}$.

Junto a estos obstáculos institucionales, el repertorio de acción del movimiento sindical se vio afectado por la creciente dificultad de las organizaciones para reclutar trabajadores y mantener la unidad. Entre 1970 y 1999, la tasa promedio de sindicalización cayó en más de la mitad. Si antes del golpe de Estado se estima que más del $30 \%$ de los trabajadores pertenecía a un sindicato ${ }^{22}$, esta cifra descendía a $14 \%$ a finales de la década del $1990^{23}$. Si bien durante los 2000, la tendencia se invirtió, la tasa continuó siendo mucho menor que en la década del 1970 (un $17 \%$ en el 2014) ${ }^{24}$. Por otra parte, el movimiento sufrió una creciente fragmentación en todos sus niveles. Incluso la CUT que había logrado históricamente afirmar su unidad, sufrió divisiones que dieron nacimiento a dos nuevas centrales sindicales: la Unión Nacional de Trabajadores (UNT) y la Central Autónoma de Trabajadores $^{25}$.

Los cambios institucionales y la debilidad interna tuvieron un claro impacto en las estrategias de los sindicatos en todos sus niveles. A nivel de las empresas, la huelga se transformó en una medida de último recurso, sino en una acción descartada. En efecto, el número de huelgas disminuyó de 247 en 1992 a 174 en el $2010^{26}$. Si bien la negociación se asentó al interior de las empresas más grandes, su cobertura siguió siendo baja. Si en 1992

20 A estos dos procesos de reformas laborales hay que sumar la Reforma al Código del Trabajo introducida por la promulgación de la Ley $\mathrm{N}^{\circ} 20940$ en 2016, cuyo impacto no ha sido considerado en el presente análisis.

21 Para un análisis detallado de las políticas laborales de la Concertación, ver SEHNBRUCH, Kirsten. The Chilean Labor Market: A Key to Understanding Latin American Labor Markets, Annotated edition, New York: Palgrave Macmillan, 2006; Cook, Maria Lorena. The Politics of Labor Reform in Latin America: Between Flexibility and Rights. University Park, Pa.: Penn State University Press, 2008; WinN, Peter. The Pinochet era. En Winn, P. (Ed.) Victims of the Chilean miracle: workers and neoliberalism in the Pinochet era, 1973-2002, Durham, USA: Duke University Press, 2004, p.14-70; FRANK, Volker. "Politics without Policy: The Failure of Social Concertation in Democratic Chile, 1990-2000". En Winn, P. Victims of the Chilean miracle: workers and neoliberalism in the Pinochet era, 19732002, Durham, USA: Duke University Press, 2004, p.71-126; HAAGH, Louise. Citizenship, Labour Markets and Democratization: Chile and the Modern Sequence, Palgrave Macmillan UK, 2002.

22 GARCÉs, Mario y Pedro Milos. Foch Ctch Cut: Las centrales unitarias en la historia del sindicalismo chileno, Santiago, Chile: ECO, 1988

23 Dirección Del Trabajo. Compendio estadístico de 1990 a 2014, Santiago, Chile: Dirección del Trabajo, Gobierno de Chile, 2015.

24 Idem.

25 Frías FernándeZ, Patricio. Los desafíos del sindicalismo en los inicios del siglo XXI, Becas de Investigación, Buenos Aires: CLACSO, 2008.

26 Dirección DEL TRABAJO, 2015, op. cit. 
sólo el $12 \%$ de los trabajadores asalariados estaba cubierto por un contrato o convenio colectivo, esta cifra era aún más baja en el $2013(8,4 \%)^{27}$.

El repertorio de acción de la CUT también experimentó un cambio. Hasta finales de los 1990, la CUT optó por una estrategia focalizada en la negociación con los representantes del gobierno y el mundo empresarial. Con el patrocinio y la participación de los primeros gobiernos de la Concertación, la principal central sindical apostó por alcanzar acuerdos generales con la Confederación de la Producción y del Comercio para asegurar la estabilidad de la democracia conquistada. Abandonando su tradición más revolucionaria, la CUT aceptaba el modelo de mercado con la condición de que se restituyeran los derechos perdidos y se mejoraran las condiciones de los trabajadores. Como resultado de este proceso, se firmaron una serie de "Acuerdos Marco" que incluyeron propuestas para la legislación laboral lo suficientemente vagas para evitar el conflicto entre las dos partes. Si bien los acuerdos incluyeron importantes beneficios como el aumento de las pensiones, los subsidios familiares y el salario mínimo, tuvieron escaso impacto en la legislación. Este resultado llevó con el tiempo a la CUT a endurecer su posición y a llamar a los trabajadores a movilizarse en paralelo a las negociaciones ${ }^{28}$. Pero los tiempos en que el llamado de la CUT a la movilización generaba una respuesta masiva y paralizaba el país habían quedado atrás. La central ya no gozaba de la legitimidad y del poder de convocatoria de antaño. No sólo contaba con una menor masa de trabajadores sindicalizados, sino también con un menor número de sindicatos afiliados ${ }^{29}$.

Las dificultades hicieron que los sindicatos tuvieran que ser creativos, buscaran nuevas formas de enfrentar a los empresarios y defender los intereses de los trabajadores. En esta búsqueda, algunos sindicatos se han inclinado por desafiar la ley. Un caso paradigmático de este tipo de sindicatos son las organizaciones de trabajadores subcontratados de la minería, la industria forestal y la salmonera, las que han obligado a las empresas mandantes a negociar con ellas directamente a pesar de que este tipo de negociación, al menos hasta el $2016^{30}$, no tenía respaldo legal. Para lograr esto, estas organizaciones han usado una amplia gama de estrategias que pueden ser consideradas disruptivas, como la huelga extra-legal, el bloqueo de calles o la toma de las faenas ${ }^{31}$.

Pero quizás uno de los cambios más visibles y transversales en el repertorio de acción de los sindicatos ha sido la importancia creciente de la movilización legal. Los sindicatos

27 Durán Sanhueza, Gonzalo y Marco Kremerman Strajilevich. Sindicatos y Negociación Colectiva. Panorama Estadístico Nacional y Evidencia Comparada, Santiago, Chile: Fundación Sol, 2011.

28 FRÍAS FERNÁNDEZ, 2008, op. cit.

29 Según la Encuesta Laboral (ENCLA), menos de un tercio de los sindicatos de empresa pertenecen a una central sindical, véase DiRECCIÓn DEL TrabAJO. Encuesta Laboral (ENCLA) 2011, Santiago de Chile: Dirección del Trabajo, Gobierno de Chile, 2012.

30 El 2016 fue el año en que se promulgó la Ley de subcontratación, en respuesta a las demandas de este sector por mejores condiciones laborales.

31 Aravena Carrasco, Antonio y Daniel Núñez. El Renacer De La Huelga Obrera En Chile: El Movimiento Sindical En La Primera Década Del Siglo XXI, Santiago de Chile, Instituto de Ciencias Alejandro Lipschutz, 2009. 
recurren con cada vez más frecuencia a la DT y a los tribunales para denunciar a la empresa por infracciones a la ley. Como veremos más adelante, los sindicatos no denuncian a la empresa sólo para defender un derecho que ha sido violado. Lo hacen también para ampliar su margen de maniobra en la empresa, mejorar las perspectivas de éxito durante la negociación o, incluso, para incidir en las políticas públicas. En otras palabras, los sindicatos han transformado la movilización legal en una estrategia sustitutiva o complementaria de las acciones más tradicionales.

Las cifras mostradas por la Encuesta Laboral (ENCLA) son ilustrativas del peso que tiene la movilización legal en el repertorio de acción de los sindicatos de empresa. Según esta encuesta, en el 2014, el $45,1 \%$ de los sindicatos de empresa recurrió a la DT o a los tribunales durante el año ${ }^{32}$, mientras que sólo un $14,5 \%$ realizó una huelga ${ }^{33}$. Si bien no existen datos administrativos históricos que permitan evaluar con precisión cómo ha evolucionado el peso de cada una de estas estrategias desde 1990, existente evidencia que sugiere que la estrategia legal se ha vuelto más importante desde esa fecha. Por un lado, según el Anuario estadístico de la DT, el número de denuncias presentadas por los trabajadores que han terminado en una fiscalización a la empresa han aumentado de 31.840 a 89.224 entre 1992 y el $2012^{34}$. Sólo desde el 2006, la DT tiene registro si la denuncia es presentada o no por el sindicato y, desde esa fecha, el registro señala un aumento de las denuncias interpuestas por estas organizaciones (de 6.438 a 7.910 el 2012) ${ }^{35}$. Por otro lado, los datos del Instituto Nacional de Estadísticas muestran que el número de causas laborales ingresadas ha aumentado desde que la Reforma Procesal Laboral, promulgada en 2005, se implementara en la Región Metropolitana en el 2008 (de 221.720 en el 2009 a 316.869 en $\left.2015^{36}\right)^{37}$. Lamentablemente, la información no distingue si estas causas son presentadas o no por los sindicatos.

La movilización legal también se ha vuelto parte del repertorio de la CUT. La central sindical ha emprendido campañas judiciales para intentar incidir en las decisiones del Legislativo e impulsar reformas que han sido bloqueadas por la oposición en el Parlamento. Por ejemplo, en el 2005, la CUT interpuso una demanda contra las Administradoras de Fondos de Pensiones(AFP), acusándolas de publicidad engañosa e incumplimiento de contrato, con el objetivo explícito de castigar a estas empresas y a las autoridades por las promesas incumplidas que hicieron cuando se privatizó el sistema de pensiones en los años $1980 \mathrm{~s}^{38}$. La acusación fue llevada a la Corte Suprema en el 2008 pero no prosperó. De la misma manera, la CUT emprendió una acción judicial en contra del Estado Chileno ante la

32 Este porcentaje es calculado por los autores, tras la recodificación de la pregunta 13 de la ENCLA.

33 Dirección Del Trabajo. Encuesta laboral (ENCLA) 2014. Santiago, Chile: Dirección del Trabajo, Gobierno de Chile, 2014.

34 DireCCIÓN DEL TRABAJo, 2015, op. cit.

35 Estos datos fueron solicitados directamente al Departamento de Estudios de la DT, a través del sistema de solicitud de información por transparencia, en julio del 2014.

36 Excluye datos sobre litigios respecto de cotizaciones impagas.

37 Instituto Nacional de EstadístiCAs DE Chile. Informe Anual de Justicia, Santiago, Chile: INE, 2016.

38 LA NACIÓN. CUT anuncia acciones legales contra AFPs por publicidad engañosa, La Nación, abril 24, 2005a. 
Corte Interamericana de Derechos Humanos con el fin de eliminar la prohibición existente sobre los dirigentes sindicales para ocupar cargos públicos en paralelo a los cargos sindicales ${ }^{39}$.

Así como las limitaciones impuestas por la ley han debilitado la capacidad de los sindicatos de recurrir con éxito a la huelga, el fortalecimiento de las instituciones arbitradoras durante los gobiernos de la Concertación ha permitido que los trabajadores recurran cada vez más a la movilización legal. En efecto, las autoridades militares intervinieron las IAS y debilitaron su capacidad de velar por el cumplimiento de los derechos laborales. La situación de la justicia laboral llegó a tal punto que expertos afirmaban a comienzos de los noventa que los trabajadores chilenos no gozaban de una verdadera garantía para defenderse contra de los abusos empresariales ${ }^{40}$. La situación cambió significativamente luego de la recuperación de la democracia. La DT experimentó una incesante multiplicación de sus funciones. Casi todos los nuevos estatutos laborales fueron acompañados por una referencia a esta agencia administrativa, nuevas funciones y recursos $^{41}$ (Ugarte 2008). De la misma manera, la Reforma Procesal Laboral simplificó considerablemente los procesos, amplió el número de tribunales y de jueces laborales, entre otras medidas que acortaron los tiempos de las causas de años a unos pocos meses.

En suma, existe abundante evidencia que la movilización legal se ha transformado en una estrategia clave para el movimiento sindical. ¿Qué efectos ha tenido este cambio en el repertorio para los trabajadores?

\section{METODOLOGÍA}

\section{Datos}

Para responder la pregunta de investigación utilizamos dos tipos de fuentes. En primer lugar, entrevistas semi-estructuradas a 47 dirigentes sindicales de distintas estructuras sindicales, afiliadas y no afiliadas a la Central Unitaria de Trabajadores; 3 abogados de empresa; 2 abogados de trabajadores; 1 juez y 10 funcionarios de la DT. Todas las entrevistas fueron conducidas en Santiago entre el 2009 y el 2016, duraron entre 40 y 200 minutos, fueron registradas en audio y trascritas integralmente. En segundo lugar, se revisaron y sistematizaron datos administrativos. Específicamente los compendios estadísticos de la DT, los Anuarios y los fallos judiciales publicados por el Poder Judicial.

Análisis

El análisis de datos se realizó en tres etapas. Primero se realizó un análisis temático y

39 LA NACión. CUT recurre a la Corte Interamericana para acceder al Congreso, La Nación, mayo 17, 2005b

40 Gazmuri, Consuelo. "La reforma a la justicia laboral. Contenidos, implicancias y perspectivas para una modernización de las relaciones laborales", en Mitos y realidades del mercado laboral en Chile, editado por Jaime Ensignia, Santiago de Chile: Fundación Friedrich Ebert, 2005.

41 Ugarte, José Luis. "Inspección del trabajo en Chile: vicisitudes y desafíos", Revista Latinoamericana de Derecho Social, 6:Enero 2008, p.187-204. 
categorial de las entrevistas ${ }^{42}$. Mediante codificación abierta se identificaron los temas prominentes entre los participantes, los cuales luego fueron organizados considerando las preguntas de investigación y marco teórico. Segundo se complementó el análisis cualitativo con datos administrativos. Finalmente se realizó un análisis relacional de los temas y categorías emergentes $^{43}$.

\section{RESULTADOS}

Frente a la pregunta por los efectos que ha tenido la acción legal para los trabajadores, los entrevistados hacen referencia a dos temas que pueden ser analíticamente distinguidos. Por un lado, el impacto que tienen las campañas exitosas antes las IAs, tanto en los derechos de los trabajadores como en la fuerza de sus organizaciones. Por otro lado, las posibilidades de que estas campañas resulten exitosas. Esto último remite al problema de la sensibilidad que tienen las IAs a la causa de los trabajadores.

\section{Una estrategia con un impacto ambivalente}

Los actores entrevistados identifican consecuencias tanto en el ámbito del derecho como en el de la organización sindical. La Tabla 1 resume estos efectos, según si son considerados positivos o negativos.

Tabla 1: Resultados de la intervención de las IAs para los trabajadores

\begin{tabular}{|c|c|c|}
\hline Ámbito $^{\text {Valor }}$ & Positivo & Negativo \\
\hline Derechos & $\begin{array}{l}\text { - Debilitamiento de una } \\
\text { lógica de acumulación } \\
\text { basada en la transgresión de } \\
\text { la norma } \\
\text { - Ampliación "de hecho" de } \\
\text { ciertos derechos } \\
\text { - Presión para la creación de } \\
\text { nuevas leyes }\end{array}$ & $\begin{array}{l}\text { - Confirmación de la } \\
\text { inoperancia de las leyes } \\
\text { - Disminución del impacto } \\
\text { de ciertas leyes en las } \\
\text { condiciones de } \\
\text { trabajadores los }\end{array}$ \\
\hline $\begin{array}{l}\text { Organización } \\
\text { sindical }\end{array}$ & $\begin{array}{l}\text { - Ampliación del poder de } \\
\text { negociación de los } \\
\text { sindicatos en las empresas } \\
\text { - Ampliación de las bases y } \\
\text { fidelización de los }\end{array}$ & 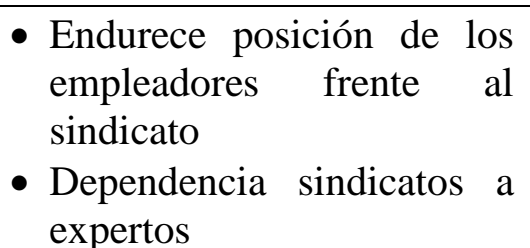 \\
\hline
\end{tabular}

42 Charmaz, Kathy. "Grounded theory", en R. M. Emerson (Ed.), Contemporary field research, Long Grove, IL, Waveland, 2001, p.335-352.

43 Clarke, Adele E. y Susan Leigh Star. "The social worlds framework: A theory/methods package", en Hackett, E., Amsterdamska, O., Lynch, M., \& Wajcman, J. (Eds.), The handbook of science and technology studies, Cambridge, Massachusetts: The MIT Press, 2008, p.113-139. 


\begin{tabular}{l|l|l}
\hline trabajadores a los sindicatos & $\begin{array}{l}\text { - Pérdida de credibilidad de } \\
\text { los sindicatos en las bases } \\
\text { - Empobrecimiento del } \\
\end{array}$ \\
& $\begin{array}{l}\text { compromiso de las bases } \\
\text { con la organización }\end{array}$ \\
\hline
\end{tabular}

Fuente: Elaboración de los autores en base a entrevistas

\section{Cambios en los derechos}

Los entrevistados reconocen que la sola defensa de los derechos existentes, a través de la sanción de las empresas, constituye una manera de ampliar el margen de acción de los trabajadores pues, en muchas empresas, la infracción de la normativa laboral es la regla y no la excepción. Por esta razón, hacer respetar los derechos existentes se interpreta como un combate en contra de "un capitalismo mercantilista" que funda la acumulación en la explotación radical de los trabajadores ${ }^{44}$.

Pero la sanción por parte de las IAs no siempre provoca un cambio en las prácticas de las empresas. Esto es particularmente cierto cuando interviene la DT pues, como lo señalan varios dirigentes, las multas que impone este organismo no siempre son suficientes para compensar las ganancias que obtiene el empleador transgrediendo las normas. En estos casos, "la multa no significa nada para el trabajador" 45 y las normas son percibidas como inoperantes.

Sin perjuicio de lo anterior, los entrevistados reconocen que las campañas ante las IAs han resultado ser un mecanismo efectivo para lograr ciertas prerrogativas que la ley no entrega de manera evidente y que, por lo mismo, han sido negadas en la práctica por las empresas con el conocimiento del Poder político. Un ejemplo que dan de esto fue lo que sucedió con la negociación colectiva inter-empresa durante la última década. Según los actores, antes que fuera promulgada la llamada "ley del multirut" (Ley No 20760, 2014), los sindicatos lograron que las IAs obligaran a las empresas del mismo dueño a negociar con los sindicatos de manera conjunta a pesar que la norma era ambigua al respecto y justificaba que los empresarios dividieran las empresas para eludir obligaciones laborales como la mencionada. En la misma línea, subrayan que, en el 2015, la Corte Suprema emitió un fallo que cambió la interpretación del artículo 381 del Código a favor de los trabajadores puesto que estableció que la sustitución de los trabajadores en huelga con personal interno también debía ser considerada como reemplazo. La ley no hace mención explícita a este tipo de práctica y, por lo tanto, permitía hasta este fallo que las empresas recurrieran a ella eludiendo las restricciones que establece el Código para el reemplazo con trabajadores externos (Causa $\left.\mathrm{N}^{\mathrm{0}} 15293-2014\right)^{46}$. El fallo habría presionado al gobierno para que

44 Dirigente sindicato de empresa, sector bancario; local del sindicato, diciembre 2009

45 Dirigente Federación nacional, sector sanitario; local del sindicato, noviembre 2014.

46 Rol 15293/2014, resolución n ${ }^{\circ} 68497$ de Corte Suprema. Fallo sanciona con una multa de 10 UTM, a beneficio del 
incorporara esta modificación en el proyecto de reforma laboral presentado al Parlamento a fines de ese año. Como en estos casos, la mayor parte de las leyes promulgadas en materia laboral que han beneficiado a los trabajadores desde 1990 han sido antecedidas por dictámenes de la DT o sentencias judiciales ${ }^{47}$.

De lo anterior se sigue que las IAs no solo han generado "derechos de hecho" para los trabajadores, también han ejercido presión en el Poder político para que este promulgue leyes que formalicen estos nuevos derechos. Las entrevistas permiten distinguir dos mecanismos mediante los cuales se produce esta presión. En primer lugar, el lobby que realizan los propios directores de la DT ante autoridades y representantes del Poder legislativo. Como señala uno de los funcionarios de la DT: "[...] el director va a la Cámara o al Senado, o el director va y propone algo a la Moneda o pregunta aquí en el Ministerio del trabajo. Ese es un lobby institucional"48. El segundo mecanismo es a través de la presión que ejercen los propios dirigentes sindicales. Los sindicatos utilizan los dictámenes y las sentencias de tribunales para dar solidez a sus demandas. Como lo señala una de los entrevistados: "[...] [los dictámenes] nos dieron como el sustento legal, entre comillas, para poder decir que en realidad había un problema aqui [...] Y, entonces había ese sustento legal para ir a reclamar, y ahi después empezaron a utilizar al congreso. "49

\section{Cambios en la organización sindical}

Los entrevistados también reconocen que el recurso a las IAs tiene también consecuencias extra-jurídicas para los trabajadores. Una de ellas, son los cambios que se producen en la relación de los sindicatos con el empleador. Los funcionarios de la DT señalan que los sindicatos denuncian a las empresas con más frecuencia en los periodos de negociación colectiva "[...] como diciendo, somos capaces de cualquier cosa" ${ }^{\text {"50 }}$. De esta manera, los sindicatos buscan presionar al empleador para que se muestre más sensible a sus demandas durante la negociación. Las organizaciones de trabajadores utilizan el recurso de manera táctica, "guardando" la denuncia de las infracciones para momentos donde requieren ejercer mayor fuerza contra la empresa ${ }^{51}$.

Sin embargo, esta estrategia puede tener resultados no deseados como que el sindicato "pierda la confianza de la empresa" 52. El empleador "queda con la vena con los trabajadores" $" 53$ y puede verse tentado a tomar medidas en contra de los sindicatos o de los

Servicio Nacional de Capacitación y Empleo, a la empresa RGM Mallas de Alambre Ltda. y al pago de las costas de la causa por "práctica desleal en la negociación colectiva", durante una huelga legal, en octubre de 2013.

47 Juez, Primer Juzgado de Letras del Trabajo de Santiago; oficina juez; agosto 2014.

48 Abogado del Departamento Jurídico de la DT; local de la DT, Santiago centro, julio 2014.

49 Tesorera sindicato de empresa, compañía transporte aéreo; oficina investigadora principal, octubre 2014.

50 Fiscalizador y receptor de denuncias sindicales; local de la DT zona Poniente en Región Metropolitana; agosto 2014.

51 Fiscalizadora; local de la DT zona Oriente en Región Metropolitana; agosto 2014

52 Dirigente Central Unitaria de Trabajadores (CUT); oficina dirigente, noviembre 2014

53 Dirigente Federación nacional, sector sanitario; local del sindicato, noviembre 2014. 
trabajadores involucrados en las campañas. Asimismo, puede afectar la fuerza de la organización, sobre todo cuando se transforma en la única forma de confrontación. Por ejemplo, puede generar una dependencia excesiva a abogados o asesores externos. Denunciar a las empresas ante las IAs y, en particular, ante los tribunales exige de un conocimiento y experticia técnica que los dirigentes sindicales rara vez poseen. Los profesionales externos que los sindicatos contratan para denunciar a las empresas intervienen muchas veces más de la cuenta en las decisiones de la organización. En palabras de una entrevistada: "[...] hacen lo que quieren con las organizaciones, manipulan a los dirigentes y al final los sindicatos son de ellos, no son ni de los dirigentes ni de las bases" $" 54$.

Otra consecuencia no deseada de la estrategia legal es el cambio en la relación de las organizaciones con sus bases. Los dirigentes sindicales entrevistados son conscientes que los trabajadores desarrollan un mayor interés en afiliarse al sindicato cuando tienen éxito en las campañas ante la DT o los tribunales. Por esta razón, utilizan los triunfos que obtienen ante las IAs como enganche para atraer nuevos miembros a la organización, fidelizar a los socios existentes o ser reelegidos. Pero los dirigentes reconocen que esta misma estrategia puede generar un problema, pues acostumbra a los trabajadores a exigir de los sindicatos un servicio en vez de instarlos a participar activamente por una causa común. En los términos de una de las dirigentes entrevistadas: "[...]los sindicatos se ven más bien como un grupo de personas que están ahí para ayudarte, para resolverte los problemas, para tomarte de la manito y llevarte a reclamar, para no dar la cara tú sino que la dé otro por ti. "55 Sin capacidad para movilizar a los trabajadores en acciones de protesta que impliquen más riesgos, los sindicatos pueden tener dificultades para lograr cambios más sustanciales.

\section{Resultados susceptibles a los ciclos políticos}

Pero las campañas frente a la DT no siempre terminan en una multa a la empresa o la acción judicial en una sanción al empleador. Los entrevistados subrayan que este resultado depende en gran medida de la sensibilidad del juez o las autoridades de la DT respecto de la causa de los trabajadores. Los entrevistados identifican diferencias importantes en este plano, tanto entre las distintas IAs como en la conducta de cada una a lo largo del tiempo.

\section{La Dirección del Trabajo}

Los entrevistados coinciden en que la DT ha sido un agente defensor de los trabajadores desde la recuperación de la democracia en 1990, aunque reconocen que ha sido muy susceptible a los cambios políticos. El primer giro importante que identifican ocurrió tras la renuncia de María Ester Feres, abogada socialista que estuvo a la cabeza de la institución entre 1994 y el 2004, quien infundió a la agencia una marcada inclinación ideológica en

54 Tesorera sindicato de empresa, compañía transporte aéreo; oficina investigadora principal, octubre 2014.

55 Tesorera sindicato de empresa, compañía transporte aéreo; oficina investigadora principal, octubre 2014. 
favor de los trabajadores. Como lo señala uno de los abogados de empresa: "Hasta la caída del gobierno militar, la DT era un buzón que no servía para mucho a los trabajadores y donde los empleadores eran atendidos como gente importante, digna de todo respeto y de toda credibilidad. Y empezó el ciclo de gobiernos democráticos y la DT se empezó a cargar cada vez más a la izquierda [...] y los empleadores comenzaron a ser tratados como delincuentes [...] Un hito en esta procesión lo marca María Estar Feres[...]"156

Para los entrevistados, la renuncia de Feres generó un proceso progresivo de neutralización de la DT que habría llegado a un punto culminante tras el triunfo de la derecha en las elecciones presidenciales del 2009. Como señala uno de los dirigentes, durante el gobierno de Sebastián Piñera "Hubo un decaimiento y la verdad de las cosas que se aburguesaron más de lo que estaban en la DT"57.

Los datos administrativos confirman esta tendencia. Como muestra el Gráfico 1, el número de fiscalizaciones realizadas por la DT se multiplicó significativamente entre 1990 y el 2007 para luego estancarse. Asimismo, el porcentaje de estas fiscalizaciones que terminó en una multa contra la empresa disminuyó considerablemente durante el mismo periodo y, en especial, desde los últimos años de la administración de Feres.

Gráfico 1: Fiscalizaciones y tasa de multas entre 1990 y $2014 *$

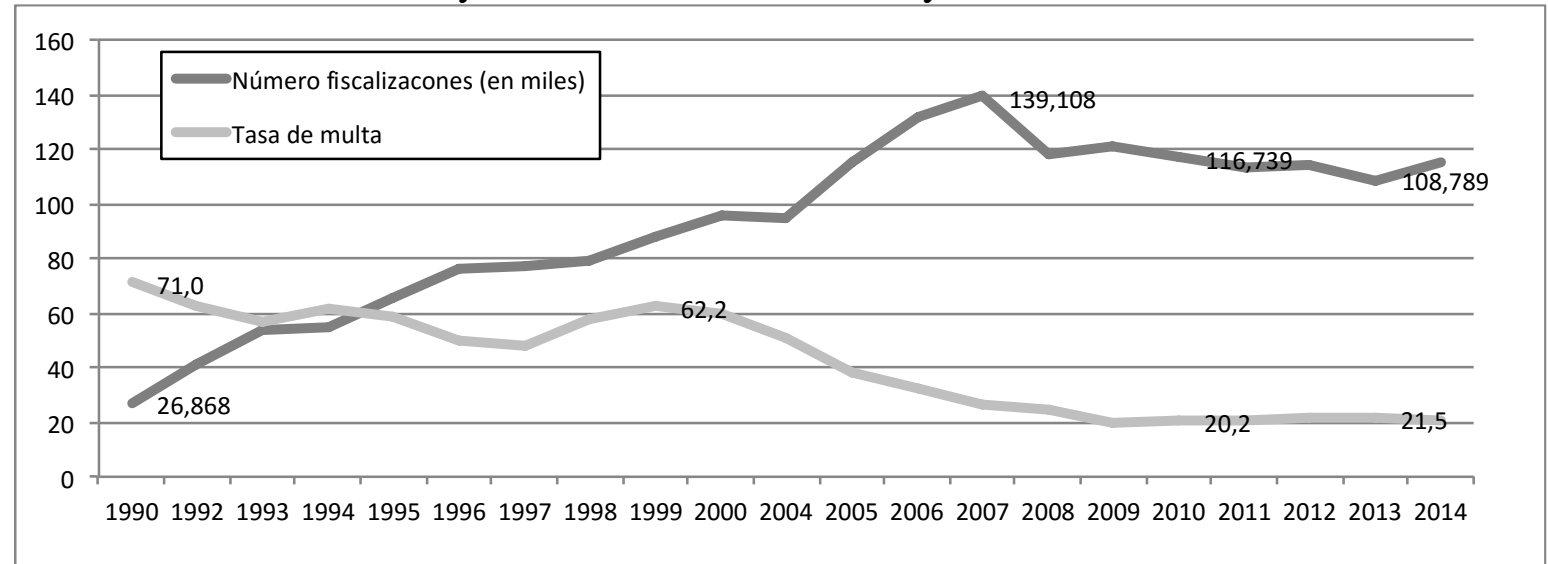

Fuente: Compendio estadístico, DT, 2014. *No existen datos sobre las multas cursadas para 1991, 2001, 2002 y 2003.

Los funcionarios de la DT señalan que la caída de la tasa de multas se explica, en gran parte, por un proceso de "modernización" precisa de las prerrogativas que tienen sus funcionarios durante las fiscalizaciones en las empresas; la simplificación de los procedimientos para que las empresas reclamen las

56 Abogado laboral de empresas; oficina privada, agosto 2014.

57 Presidente sindicato inter-empresa de sector transporte; local del sindicato; octubre 2014.

58 Abogado laboral de empresas y trabajadores; oficina investigadora principal, agosto 2014. 
multas; y la restricción del margen de acción de la agencia para interpretar la ley. Esta modernización habría sido una respuesta a la presión ejercida por las propias empresas para revertir las sanciones de la DT en las Cortes a lo largo de los años. Como señala uno de los abogados entrevistados: "Con el antiguo procedimiento [antes de la Reforma Procesal Laboral], las Cortes de apelaciones echaban abajo más del $70 \%$ de las multas de la DT(... $)^{\text {"59 }}$. Por otra parte, hasta el 2014, la Corte Suprema "le ha recortado muchas de las facultades [a la DT]" 60 ratificando la posición de los empleadores según la cual los inspectores de la DT no tienen las prerrogativas para fiscalizar situaciones complejas o interpretar los hechos, lo que sería atribución de los tribunales ${ }^{61}$. Estos resultados obligaron a la DT a modificar sus prácticas.

A pesar de lo anterior, los empleadores continúan acusando a la DT de representar los intereses de los trabajadores: "Hay un sesgo en favor del trabajador [...] Uno esperaría un trato objetivo y eso, de repente, se pierde"

\section{Los tribunales laborales}

Por otra parte, los entrevistados coinciden en que los jueces de los tribunales laborales tienden a tener una actitud "pro-trabajadores" 63 y algunos postulan incluso que actúan con sesgo ideológico en contra de las empresas. En palabras de un abogado de empresas: "En los tribunales laborales tú no tienes la más mínima garantía de ecuanimidad"64.

Los datos de la Defensoría Pública refuerzan esta imagen pues muestran que los casos que ingresan a tribunales tienden mayoritariamente a resolverse en beneficio del trabajador. Por ejemplo, en el 2013, el 48,1\% de las causas ingresadas a tribunales por la Defensoría terminaron en un acuerdo con el empleador; un $34,5 \%$ en un veredicto completamente favorable al o los trabajadores; y sólo un $2 \%$ parcialmente o nada favorable al o los trabajadores $^{65}$ (Ministerio de Justicia 2013).

Pero la acción en tribunales no fue siempre tan favorable a los trabajadores. Antes de la Reforma Procesal Laboral del 2005, los litigios eran muy largos y los procedimientos complejos. La Reforma "dio vuelta la tortilla" "66 pues, no sólo redujo los tiempos de las causas, sino también gatilló una transformación en la cultura y las prácticas de los jueces que habría favorecido a los trabajadores. Por ejemplo, los tribunales se habrían vuelto más sensibles a los dictámenes de la DT que representan los intereses de los trabajadores ${ }^{67}$.

\footnotetext{
59 Abogado laboral de empresas; oficina privada; agosto 2014.

60 Juez, Primer Juzgado de Letras del Trabajo de Santiago; oficina juez; agosto 2014

61 Juez, Primer Juzgado de Letras del Trabajo de Santiago; oficina juez; agosto 2014

62 Abogado laboral de empresas y trabajadores; oficina investigadora principal, agosto 2014.

63 Juez, Primer Juzgado de Letras del Trabajo de Santiago; oficina juez, agosto 2014.

64 Abogado laboral de empresas; oficina privada; agosto 2014.

65 El 15,4\% de las causas restantes es clasificado de "Otros términos" por el Anuario citado.

66 Abogado laboral de empresas; oficina privada; agosto 2014.

67 Abogado laboral de empresas; oficina privada, agosto 2014.
} 
Asimismo, el nuevo sistema hizo más difícil que las empresas anularan las multas de la DT pues el reclamo se realiza en única instancia ${ }^{68}$.

\section{La Corte Suprema}

Según los entrevistados, la sensibilidad de los tribunales a la causa de los trabajadores se habría fortalecido con los cambios recientes en la Corte Suprema. Tradicionalmente, la Corte Suprema ha sido "bastante más pro empleador" 69 que los tribunales especializados. Sin embargo, esta situación se invirtió el 2014 tras el cambio en la composición de la Corte. En el sistema chileno, son los miembros de la Corte quienes proponen los nombres de los nuevos magistrados, pero es el presidente quien decide entre los candidatos, y los parlamentarios quienes ratifican esta decisión. En abril del 2014, la presidenta Michelle Bachelet nombró a Carlos Cerda Fernández como nuevo ministro de la Corte. La integración de este conocido defensor de los derechos humanos y la salida de Patricio Valdés alteró el equilibrio de poder al interior de la Sala encargada de las causas laborales en beneficio de los magistrados "fuertemente pro laborales"70.

Tabla 1: Frecuencia de Recursos de Unificación de Jurisprudencia según tipo y sector de recurrente

\begin{tabular}{|c|c|c|c|c|c|c|c|c|c|c|c|c|}
\hline & \multicolumn{9}{|c|}{ Empleadores } & \multirow{2}{*}{\multicolumn{3}{|c|}{$\begin{array}{c}\text { Trabajadores } \\
\text { General }\end{array}$}} \\
\hline & \multicolumn{3}{|c|}{ Público } & \multicolumn{3}{|c|}{ Privado } & \multicolumn{3}{|c|}{ General } & & & \\
\hline & $T$ & A & $\%$ & $T$ & $\mathrm{~A}$ & $\%$ & $T$ & $\mathrm{~A}$ & $\%$ & $\mathrm{~T}$ & $\mathrm{~A}$ & $\%$ \\
\hline 2009 & 2 & 2 & 100 & 6 & 0 & 0 & 8 & 2 & 25 & 2 & 2 & 100 \\
\hline 2010 & 42 & 38 & 91 & 30 & 9 & 30 & 72 & 47 & 65 & 22 & 0 & 0 \\
\hline 2011 & 16 & 5 & 31 & 48 & 26 & 54 & 64 & 31 & 48 & 20 & 2 & 10 \\
\hline 2012 & 19 & 16 & 84 & 39 & 27 & 69 & 58 & 43 & 74 & 16 & 0 & 0 \\
\hline 2013 & 19 & 13 & 68 & 112 & 50 & 45 & 131 & 63 & 48 & 40 & 7 & 18 \\
\hline 2014 & 31 & 2 & 7 & 13 & 0 & 0 & 44 & 2 & 5 & 29 & 20 & 69 \\
\hline
\end{tabular}

Fuente: Elaborado por los autores en base a análisis de fallos judiciales (Poder Judicial, 2015) *T: Total de REUs presentados; A: Total de REUs acogidos por la Corte; \%: porcentaje de REUs acogidos

El análisis de Recursos de Unificación de Jurisprudencia (REUs) ${ }^{71}$ presentados ante la Corte Suprema confirma las observaciones anteriores. Como se observa en la Tabla 1, entre el 2010 y el 2013, la Corte acogió un porcentaje bajo de los recursos interpuestos por los trabajadores, mientras que aceptó más de la mitad de las acciones interpuestas por los

68 Abogado laboral de empresas; oficina privada, agosto 2014.

69 Abogado laboral de empresas y trabajadores; oficina investigadora principal, agosto 2014

70 Abogado, representante de la Asociación Gremial de Abogados Laboralistas; oficina privada, agosto 2014.

71 El Recurso de Unificación de Jurisprudencia (REU) es la acción con la que cuentan las empresas y trabajadores para anular las sentencias de instancias anteriores ante la Corte Suprema. En esta acción, el recurrente debe demostrar que existen sentencias que interpretan la ley de manera diferente a la utilizada por el juez en su causa y demandan a los magistrados que unifiquen el criterio. Cuando la Corte acoge el recurso interpuesto, da la razón al recurrente y establece una interpretación oficial de la ley en cuestión. 
empleadores, especialmente de los del sector público (empresas estatales, municipalidades o instituciones públicas). Esta tendencia se invirtió radicalmente en el 2014, año en que la Corte acogió casi el $70 \%$ de los REU presentados por trabajadores mientras que sólo un 4,5\% de los interpuestos por empleadores. Asimismo, la tabla muestra que las empresas privadas han reaccionado a este cambio de orientación, pues los recursos que interpusieron ante la Corte disminuyeron de 112 a 13 el 2014.

\section{Conclusiones}

Los resultados de la investigación sugieren que la movilización legal ha permitido a los sindicatos defender los intereses de los trabajadores en un contexto institucional donde resulta difícil emprender formas de acción más clásicas. A través de la movilización legal, los sindicatos han logrado ganar ciertos "derechos de hecho" y ampliar su margen de maniobra en las empresas.

Sin embargo, de las entrevistas a los actores se destaca que estos logros están lejos de ser revolucionarios y se identifican dos causas de esto. En primer lugar, el cambio en el repertorio de acción de las organizaciones de trabajadores ha tenido impactos no deseados que pueden entenderse bajo el campo simbólico-estratégico de los efectos de la movilización legal ${ }^{72}$, tales como el debilitamiento del compromiso de las bases sindicales y la dependencia de las organizaciones a asesores externos. Esto limita la capacidad de los sindicatos de movilizar a los trabajadores en campañas que implican un compromiso más fuerte $\mathrm{y}$, por lo tanto, pueden estar obstaculizando un cambio más sustancial del orden laboral heredado. En segundo lugar, los logros no han sido revolucionarios porque las IAs chilenas han tenido una conducta variable en el tiempo. El activismo de la DT en los 1990 tendió a desaparecer a fines de la década, mientras que el activismo de los tribunales laborales fue opacado por la posición conservadora de la Corte Suprema hasta el 2013. El giro en la orientación de esta última institución abre una nueva oportunidad para la movilización legal de los sindicatos, donde es posible esperar que los sindicatos logren nuevos "derechos de hecho". Sin embargo, este cambio de orientación es aún muy reciente para evaluar su impacto.

En suma, el artículo llama a no ser a priori ni excesivamente optimista ni tajantemente pesimista respecto a las potencialidades de la movilización legal como instrumento de presión social en las relaciona laborales. Los efectos de esta estrategia deben ser evaluados tomando en cuenta las particularidades de cada contexto y el periodo histórico. En el caso chileno, la susceptibilidad de las IAs a los ciclos políticos y la debilidad de los sindicatos han limitado, hasta ahora, el alcance de esta estrategia para los trabajadores. Sin embargo, es plausible pensar que, sin el recurso a esta estrategia, los sindicatos habrían tenido aún

72 Albiston, 2011, op. cit. 
menos capacidad de incidencia en las empresas y las políticas públicas. Sin este vuelco estratégico de los sindicatos, es razonable pensar que la situación de los trabajadores habría sido aún más precaria a lo largo de las últimas décadas. La movilización legal ha dado dinamismo la lucha sindical en un contexto donde la huelga y la negociación colectiva son alternativas institucionalmente limitadas.

Por último, el artículo invita al mundo académico a tomar en consideración el papel de la movilización legal en las relaciones laborales. Sin referencia a esta forma de acción es difícil entender cómo el movimiento sindical en países como Chile ha sobrevivido en el tiempo a pesar de los múltiples problemas que enfrenta. La literatura sobre relaciones laborales ha sido generalmente ciega a este fenómeno, tanto en Chile como en países con mayor desarrollo científico. Este artículo busca cambiar esta situación.

\section{Referencias}

Albiston, Catherine. "The Dark Side of Litigation as a Social Movement Strategy", en Iowa Law Review Bulletin, 96:941, 2011, p. 61-77.

Aravena Carrasco, Antonio y Daniel Núñez. El Renacer De La Huelga Obrera En Chile: El Movimiento Sindical En La Primera Década Del Siglo XXI, Santiago de Chile, Instituto de Ciencias Alejandro Lipschutz, 2009.

BursteIn, Paul. "Legal Mobilization as a Social Movement Tactic: The Struggle for Equal Employment Opportunity", American Journal of Sociology, 96:5, 1991, p.1201-25.

Charmaz, Kathy. “Grounded theory”, en R. M. Emerson (Ed.), Contemporary field research, Long Grove, IL, Waveland, 2001, p.335-352.

CichowsKi, Rachel A. "Women's Rights, the European Court, and Supranational Constitutionalism", en Low \& Society Review, 38:3, 2004, p.489-513.

CHEN, Feng. "Legal Mobilization by Trade Unions: The Case of Shanghai", en The China Journal, 52:Julliet, 2004, p.27-45. doi:10.2307/4127883.

Clarke, Adele E. y Susan Leigh Star. "The social worlds framework: A theory/methods package", en Hackett, E., Amsterdamska, O., Lynch, M., \& Wajcman, J. (Eds.), The handbook of science and technology studies, Cambridge, Massachusetts: The MIT Press, 2008, p.113-139.

CooK, Maria Lorena. The Politics of Labor Reform in Latin America: Between Flexibility and Rights. University Park, Pa.: Penn State University Press, 2008. 
Couso, Javier. "The Judicialization of Chilean Politics: The Rights Revolution That Never Was", en The Judicialization of Politics in Latin America, editado por Angell, Alan, Rachel Sieder, y Line Schjolden, illustrated edition, New York: Palgrave Macmillan, 2005, p.105-129.

DE FAZIO, Federico Leandro. "Judicialización de la protesta sindical en Argentina: El control de constitucionalidad como espacio de participación alternativo", en Gaceta Laboral, 19:1, 2013.

Dirección Del Trabajo. Encuesta Laboral (ENCLA) 2011. Santiago de Chile: Dirección del Trabajo. Gobierno de Chile, 2012. http://www.dt.gob.cl/documentacion/1612/w3-article98995.html.

Idem. Encuesta laboral (ENCLA) 2014. Santiago, Chile: Dirección del Trabajo, Gobierno de Chile, 2014. http://www.dt.gob.cl/documentacion/1612/w3-article-108317.html.

Idem. Compendio estadístico de 1990 a 2014. Santiago, Chile: Dirección del Trabajo. Gobierno de Chile, 2015. http://www.dt.gob.cl/documentacion/1612/articles-62614_recurso_1.pdf.

DURÁN SANHUEZA, Gonzalo y Marco Kremerman Strajilevich. Sindicatos y Negociación Colectiva. Panorama Estadístico Nacional y Evidencia Comparada, Santiago, Chile: Fundación Sol, 2015.

EPP, Charles R.. The Rights Revolution: Lawyers, Activists, and Supreme Courts in Comparative Perspective, $1^{\text {a }}$ ed., Chicago: University Of Chicago Press, 1998.

FrAnK, Volker. "Politics without Policy: The Failure of Social Concertation in Democratic Chile, 1990-2000", en Winn, P. Victims of the Chilean miracle: workers and neoliberalism in the Pinochet era, 1973-2002, Durham, USA: Duke University Press, 2004, p.71-126.

FRÍAS FERnÁNDEZ, Patricio. Los desafíos del sindicalismo en los inicios del siglo XXI, Becas de Investigación, Buenos Aires: CLACSO, 2008.

GARCÉs, Mario y Pedro Milos. Foch Ctch Cut: Las centrales unitarias en la historia del sindicalismo chileno, Santiago, Chile: ECO, 1988.

GAZMURI, Consuelo. "La reforma a la justicia laboral. Contenidos, implicancias y perspectivas para una modernización de las relaciones laborales", en Mitos y realidades del mercado laboral en Chile, editado por Jaime Ensignia, Santiago de Chile: Fundación Friedrich Ebert, 2005.

GINSBURG, Tom. "Courts and New Democracies: Recent Works", Law \& Social Inquiry 37:3, 2012, p.720-42.

HaAgh, Louise. Citizenship, Labour Markets and Democratization: Chile and the Modern Sequence. Palgrave Macmillan UK, 2002.

HANDLER, Joel F. Social Movements and the Legal System: A Theory of Law Reform and Social 
Change. New York: Academic, 1978.

Instituto Nacional de Estadísticas de Chile. Informe Anual de Justicia. Santiago, Chile: INE, 2016. www.ine.cl.

LA NACIÓN. CUT anuncia acciones legales contra AFPs por publicidad engañosa. La Nación, abril 24, 2005a. http://www.lanacion.cl/noticias/site/artic/20050424/pags/20050424132953.html.

Idem. CUT recurre a la Corte Interamericana para acceder al Congreso. La Nación, mayo 17, $2005 b$.

MCCAMMON, Holly J.. Labor's Legal Mobilization Why and When Do Workers File Unfair Labor Practices?. Work and Occupations, n 28(2), 2001, p. 143-75. doi:10.1177/0730888401028002002.

MCCANN, Michael. Rights at Work: Pay Equity Reform and the Politics of Legal Mobilization. Chicago: University of Chicago Press, 1994.

Idem. Law and Social Movements: Contemporary Perspectives. En Annual Review of Law and Social Science, n 2(1), 2006, p.17-38.

Ministerio DE JUSTICIA. Anuario Estadístico Justicia Laboral. Santiago, Chile: Ministerio de Justicia, 2013. http://historico.minjusticia.gob.cl/n2962_15-05-2014.html.

NEJAIME, Douglas. “The legal mobilzation dilemma”, Emory Law Journal, 61:32, 2011, p.663-735.

RoomKIn, Myron. "A Quantitative Study of Unfair Labor Practice Cases”. En Industrial and Labor Relations Review, 34(2), 1981, pp. 245. doi:10.2307/2522539.

Rosenberg, Gerald. The Hollow Hope: Can Courts Bring About Social Change?, Chicago: University of Chicago Press, 1991.

Rubenstein, William B. "Divided We Litigate: Addressing Disputes Among Group Members and Lawyers in Civil Rights Campaigns", The Yale Law Journal, 106:6, 1997, p.1623-1681.

Scheingold, Stuart The Politics of Rights, Lawyers, Public Policy, and Political Change. New Haven, CT: Yale Univ. Press, 1974.

SEHNBRUCH, Kirsten. The Chilean Labor Market: A Key to Understanding Latin American Labor Markets, Annotated edition, New York: Palgrave Macmillan, 2006.

SHAPIRO, Martin. Courts: A Comparative and Political Analysis. Chicago: Univ. of Chicago Press, 1981.

SIEDER, Rachel, Line Schjolden, y Adam Angell (eds). The Judicialization of Politics in Latin America. New York: Palgrave Macmillan, 2005. 
UgARTE, José Luis. "Inspección del trabajo en Chile: vicisitudes y desafíos", Revista Latinoamericana de Derecho Social, 6:Enero 2008, p.187-204.

Winn, Peter. The Pinochet era. En Winn, P. (Ed.) Victims of the Chilean miracle: workers and neoliberalism in the Pinochet era, 1973-2002, Durham, USA: Duke University Press, 2004, p. 14-70. 